\title{
émulations
}

\section{Christophe Traïni (dir.) - Émotions et expertises. Les modes de coordination des actions collectives}

\section{Vivien Gain}

Émulations - Revue de sciences sociales

2016, «Comptes rendus critiques, En ligne »

\section{Article disponible à l'adresse suivante}

https://ojs.uclouvain.be/index.php/emulations/article/view/7033

\section{Pour citer cet article}

Vivien Gain, «Christophe Traïni (dir.) - Émotions et expertises. Les modes de coordination des actions collectives », Émulations, en ligne. Mise en ligne le 15 février 2016. DOI : 10.14428/emulations.cr.007

Distribution électronique : Université catholique de Louvain (Belgique) : ojs.uclouvain.be

(C) Cet article est mis à disposition selon les termes de la Licence Creative Commons Attribution, Pas d'Utilisation Commerciale 4.0 International. http://creativecommons.org/licenses/by-nc/4.0/

Éditeur : Émulations - Revue de sciences sociales / Presses universitaires de Louvain https://ojs.uclouvain.be/index.php/emulations

ISSN électronique : 1784-5734

UCL PRESSES

UNIVERSITAIRES 


\section{Christophe Traïni (dir.) - Émotions et ex- pertises. Les modes de coordination des actions collectives}

Vivien Gain $^{1}$

Recensé : Christophe Traïni (dir.), Émotions et expertises. Les modes de coordination des actions collectives, Rennes, Presses universitaires de Rennes, 2015.

En dirigeant cet ouvrage collectif, Christophe Traïni, politologue basé à l'Institut d'études politiques d'Aix-en-Provence, franchit une étape supplémentaire de son programme général de recherche, que l'on peut tenter de résumer en une interrogation : quelle(s) place(s) accorder aux émotions dans la sociologie contemporaine et, plus précisément, dans l'étude des mouvements sociaux ? Déjà dans l'introduction d'Émotions... Mobilisation !, Traïni (2009) appelait à « se défaire d'une idée ancienne trop communément admise qui fait de l'activité politique une réflexion intellectuelle excluant l'irrationalité habituellement prêtée à l'émotion » (p. 12). Contre tout réductionnisme du fait politique comme calcul tactique ou simple prolongement d'une réflexion morale, Traïni nous encourage au contraire à nous plonger dans sa complexité, en reconnaissant que l'action collective est entre autres saturée d'émotions. Le champ d'étude de celles-ci, par contre, ne doit pas être considéré comme un champ autonome de la sociologie. Bien au contraire, les émotions se doivent d'être étudiées en relation avec le contexte et les contraintes qui les déterminent dans l'espace social. De plus, il ne faut pas oublier que les règles du jeu politique imposent aux acteurs sociaux qui s'y investissent une neutralisation des affects et une mise à l'écart de toute " passion » émotive, ainsi qu'une maitrise des connaissances comme règles de base du débat démocratique. Dès lors, ce qui importe est d'étudier la façon dont les deux pôles, celui de l'expertise et celui de l'émotion, s'articulent dans l'espace public. C'est précisément la mission que se sont donnés les multiples auteurs dans les quatre parties d'Émotions et expertises.

En tout premier lieu, lintroduction de Traïni a pour but de définir les bases théoriques qui baliseront l'approche empirique des auteurs dans les chapitres suivants. Pour explorer cette articulation entre émotions et expertises, Traïni fait appel à deux types de dispositifs de coordination : les dispositifs de sensibilisation, par lesquels il faut entendre « l'ensemble des supports matériels, des agencements d'objets, des mises en scène, que les acteurs étudiés déploient afin de susciter des réactions affectives qui

1 Étudiant en Master 2 de sociologie. 
prédisposent ceux qui les éprouvent à soutenir la cause défendue » (p. 19), et ceux de l'expertise, qui renvoie au « fait d'exciper d'une connaissance cognitive spécifique donnant droit à prescrire les normes les mieux à même de servir l'intérêt général » (p. 20). L'étude de ce couple de dispositifs en apparence antithétiques amène les chercheurs à se pencher sur un ensemble de thèmes nécessaires à la « socialisation » des émotions : quelles sont les (pré)dispositions individuelles nécessaires pour influencer ou être influencé par un dispositif de sensibilisation ? Comment considérer la relation de complémentarité asymétrique entre émotions et expertises ? Face à la complexité des contextes et à l'imprévisibilité des effets des dispositifs, comment mettre en place une étude des logiques de (dés)ajustements par rapport à ceux-ci ? Cette première définition du contexte théorique par Traïni a le mérite d'être précise tout en n’enfermant pas les différents chercheurs dans un cadre strict, leur confiant dès lors la tâche cruciale d'éviter toute conclusion théorique hâtive grâce à la grande diversité de leurs terrains ethnographiques.

Dans la première partie de l'ouvrage (sur un total de trois), intitulée « Inciter au ralliement, renforcer les loyautés », les auteurs (Karine Lamarche, Stéphanie Dechezelles et Olivier Grosjean) se penchent sur l'articulation entre des dispositifs émotionnels mis en place par des associations militantes et la façon dont ceux-ci sont réceptionnés par différents publics. On attirera particulièrement l'attention sur l'article de Lamarche, qui étudie les dispositifs de sensibilisation mis en place par l'organisation israélienne Shovrim Shtika (« Briser le silence » en hébreu), dont l’objectif est de dénoncer l’occupation par les colons juifs de terres palestiniennes en emmenant les publics faire l'expérience directe de celle-ci. Loin de se limiter à l'étude des activités mises en place, ce premier terrain permet également de souligner l'importance stratégique du ciblage des publics - qui est visé par les militants, et pourquoi ? - afin de maximiser les retombées émotionnelles et politiques, mais aussi de montrer à quel point l'étude de la biographie des activistes est nécessaire. L'alignement des militants avec leur public et/ou leur objet d'indignation leur permet de développer une expertise sur le sujet, et donc d'accroître leur capacité à éveiller chez leur public l'émotion et l'indignation qu'ils recherchent. Un autre point pertinent de ce premier chapitre est qu'il aborde également la question délicate de la position du chercheur dans son terrain ethnographique. Comment gérer sa propre implication dans les dispositifs étudiés ? Celle-ci doit-elle être recherchée, afin de comprendre effectivement les émotions qui sont évoquées ? Ou ces émotions doivent-elles être évitées à tout prix à cause de leur éventuelle influence sur l'objectivité scientifique ? Une question cruciale qui aurait peut-être mérité une plus grande attention dans les autres chapitres.

Les auteurs de la deuxième partie, « Régimes complémentaires ou concurrents ? ", se penchent plus directement sur les différentes possibilités d'articulation des dispositifs de sensibilisation et des dispositifs d'expertise dans trois cas : le procès d'Alberto Fujimori au Japon (Sophie Daviaux), les audiences publiques en Inde (Stéphanie Tawa Lama-Real) et les mobilisations contre l'extension de l'aéroport de Milan Malpensa 
(Laura Centemeri). L'analyse de Daviaux met en lumière d'une manière très efficace les allers-retours constants entre expression et mise à distance des émotions lors du procès du dictateur péruvien Alberto Fujimori. Comment l'extradition et le procès ont-ils été gérés par un tribunal national ? Comment les militants sont-ils parvenus à « prouver le caractère impérieux de leurs revendications d'impunité et de justice » (p. 96) ? Tout au long de la procédure d'extradition et de procès, la situation a oscillé entre l'invocation de fortes émotions et le rejet à tout prix de ces émotions par l'expertise juridique. D'un côté, il fut nécessaire de construire l'image d'un dictateur violent, corrompu et meurtrier, pour justifier l'extradition de Fujimori après que celui-ci se soit réfugié au Japon. De l'autre, il fallait également mettre en place un important travail de formalisation juridique de la procédure, sans quoi le procès aurait pu être considéré comme une simple vengeance politique. Lamarche décrit très bien la force du droit et de son langage procédural, permettant de contrecarrer les critiques du procès et de créer un « lieu impartial qui produit une neutralisation des disputes à travers le processus de distanciation introduit par le juge » (p. 102-103). Les émotions, cependant, furent loin d'être absentes du procès, que ce soit lors des explosions de rage du dictateur ou des audiences des victimes des massacres. De même, les dispositifs de sensibilisation mis en place par les militants pour les droits de l'homme furent primordiaux pour démocratiser l'issue du procès et inclure l'ensemble de la population péruvienne par les émotions dans ce processus de « juridicisation de l'Histoire» (p. 114). C'est en effet par le partage sur la scène publique et la reconstruction des évènements-clés de la période dictatoriale que les populations purent se réapproprier cette étape tragique de l'histoire du Pérou.

Enfin, les auteurs de la troisième partie s'intéressent aux " Procédures et stratégies d'accréditation », cette dernière étant définie par Traïni comme l'ensemble des " conduites et discours à travers lesquels des acteurs sociaux s'efforcent de présenter les qualités, les savoir-faire et les trajectoires qui leur sont propres, comme des éléments indispensables pour pouvoir énoncer le bien commun de la collectivité » (p. 23). Dans leur chapitre, Stéphane Cadiou et Maurice Olive étudient la réaction de plusieurs maires de petites communes françaises aux récentes réformes territoriales mettant en place le statut de «métropole ». Face aux experts et élites politiques critiquant leur « localisme exacerbé » ou leur " opportunisme politique », quelles sont les ressources mises en place par ces maires de petites bourgades pour prouver la légitimité de leur lutte ? C'est notamment par l'articulation des deux formes potentiellement antinomiques du proche et du public que les maires tentent de convaincre les populations. Les va-et-vient constants entre la singularité de leur commune et l'universalité de la commune leur donnent en effet une certaine forme de légitimité. Ce chapitre permet de rappeler l'importance de l'analyse biographique des acteurs, les réseaux des maires et leur attachement singulier et affectif à leur commune s'avérant des ressources de grande importance dans leur lutte.

En conclusion, le seul point qui aurait peut-être mérité plus de développements dans cet ouvrage est celui de l'étude de l'implication des chercheurs dans des terrains où ils 
font eux-mêmes l'expérience parfois directe des dispositifs qu'ils étudient. Malgré cela, Émotions et Expertises est un excellent ouvrage, ce sur au moins deux points. D'abord, sa force empirique procurée par l'impressionnant travail ethnographique des différents auteurs permet de tester en profondeur la robustesse des concepts de dispositifs de coordination et de toutes leurs possibilités d'articulation dans des contextes très variés. Ensuite, cela n'est possible que grâce à une définition précise et rigoureuse d'un cadre théorique commun, présenté de manière exemplaire par Traïni dans son introduction. Loin d'être épuisées par l'ouvrage, les relations entre dispositifs de sensibilisation et dispositifs experts semblent poser de très bonnes bases pour le futur de l'analyse de l'action politique tout en ouvrant le champ d'action de la recherche à la fois vers de nouveaux terrains ethnographiques et vers de potentiels approfondissements théoriques.

\section{Bibliographie}

TRAïNI C. (dir.) (2009), Émotions... Mobilisation !, Paris, Presses de Science Po. 